\title{
Impact of Acute Care Surgery Service on Acute Cholecystitis Management and Outcomes at a Single Academic Network
}

Godzisz S, Giles AE, Forbes S, Eskicioglu C, Nenshi R

Department of Surgery, McMaster University, Hamilton, Ontario

\section{Introduction}

- Acute cholecystitis is a common surgical disease with significant healthcare burden and cost

- Acute care surgery (ACS) model aims to address access, efficiency, and subsequent patient satisfaction

- Unclear if ACS model truly provides patient benefit in terms of pre-, intra-, and post operative care and subsequent outcomes

\section{Aim}

To determine whether ACS models provides benefit in patient outcomes and patient-centric quality care targets when compared to traditional on-call model in the setting of acute cholecystitis in the Hamilton academic hospital network

\section{Methods}

- 150 patients with a diagnosis of acute cholecystectomy and index cholecstectomy reviewed retrospectively

- Data compared between patients treated via ACS model (pooled data from two hospitals) vs on-call model (single hospital)

- Primary outcome: Overall complication rate

- Secondary outcome: Length of stay (LOS), time to operating room (TTOR)

- T-test/Mann-Whitney $U$ test for continuous data, $\chi^{2}$ / Fisher test for categorical data, logistic regression for multivariable predictors of complications

Table 1: Patient Baseline Characteristics

\begin{tabular}{|c|c|c|c|}
\hline & $\begin{array}{c}\text { Non-ACS, } \\
\mathrm{N}=50(33.3 \%)\end{array}$ & $\begin{array}{c}\text { ACS, } \\
\mathrm{N}=100(66.7 \%)\end{array}$ & $p$ \\
\hline Sex (male) & $20(40.0)$ & 39 (39.0) & 1.0 \\
\hline $\begin{array}{l}\text { Age (years), } \\
\text { mean (range) }\end{array}$ & $50.8(22-87)$ & $51.8(20-90)$ & 0.712 \\
\hline $\begin{array}{l}\text { AACCI, } \\
\text { median (range) }\end{array}$ & $1(0-7)$ & $1(0-9)$ & 0.849 \\
\hline \multicolumn{4}{|l|}{ ASA, N (\%) } \\
\hline 1 & $1(2.0)$ & $4(4.0)$ & 0.665 \\
\hline 2 & $9(18.0)$ & $32(32.0)$ & 0.105 \\
\hline 3 & $33(66.0)$ & $56(56.0)$ & 0.318 \\
\hline 4 & $7(14.0)$ & $8(8.0)$ & 0.260 \\
\hline
\end{tabular}

$\mathrm{AACCl}=$ Age-Adjusted Charlson Comorbidity Index; ASA = American Society of Anesthesiologists Score

Table 2: Perioperative Factors

\begin{tabular}{|c|c|c|c|}
\hline & $\begin{array}{c}\text { Non-ACS, } \\
\mathrm{N}=50(33.3 \%)\end{array}$ & $\begin{array}{c}A C S, \\
N=100(66.7 \%)\end{array}$ & $p$ \\
\hline $\begin{array}{l}\text { Time to admission } \\
\text { (hours), median (IQR) }\end{array}$ & $7(5-9.75)$ & $7(5-10.5)$ & 0.606 \\
\hline $\begin{array}{l}\text { Time to OR (hours), } \\
\text { median (IQR) }\end{array}$ & $\begin{array}{c}55.5(35.5- \\
70.3)\end{array}$ & $19.5(9.8-33.0)$ & $<0.001$ \\
\hline $\begin{array}{l}\text { Operative length (min), } \\
\text { median (IQR) }\end{array}$ & $\begin{array}{c}94.0(65.3- \\
114.5)\end{array}$ & $\begin{array}{c}83.0(60.0- \\
109.0)\end{array}$ & 0.466 \\
\hline Surgical approach & & & 0.161 \\
\hline Laparoscopic & $45(90.0)$ & $96(96.0)$ & \\
\hline Converted & $5(10.0)$ & $4(4.0)$ & \\
\hline $\begin{array}{l}\text { Length of Stay (days), } \\
\text { median (IQR) }\end{array}$ & $3.5(3-5)$ & $3(2-4)$ & 0.001 \\
\hline Laparoscopic & $3(3-5)$ & $2(2-4)$ & 0.003 \\
\hline Converted & $12(10-17)$ & $10.5(9.3-27.3)$ & 0.806 \\
\hline
\end{tabular}

Table 3: Complications

\begin{tabular}{lccc}
\hline & $\begin{array}{c}\text { Non-ACS, } \\
\mathbf{N}=\mathbf{5 0}(\mathbf{3 3 . 3 \% )}\end{array}$ & $\begin{array}{c}\text { ACS, } \\
\mathbf{N = 1 0 0 ~ ( 6 6 . 7 \% ) ~}\end{array}$ & $p$ \\
\hline Intraoperative & & & \\
Complications & & & \\
$\quad$ Any complication & $3(6.0)$ & $8(8.0)$ & 0.752 \\
$\quad$ Bile Duct Injury & $0(0.0)$ & $2(2.0)$ & 1.00 \\
Other Organ Injury & $1(2.0)$ & $1(1.0)$ & 1.00 \\
$\quad$ Hemorrhage & $2(4.0)$ & $5(5.0)$ & 1.00
\end{tabular}

Post operative

Complications

No

Yes (Clavien-Dindo

grade):

$35(70.0)$
$15(30.0)$
$8(16.0)$
$5(10.0)$
$1(2.0)$
$0(0.0)$
$1(2.0)$
$0(0.0)$
$0(0.0)$
$6(12.0)$
$4(8.0)$

$81(81.0)$

$19(19.0)$

$6(6.0)$

$6(6.0)$

$6(6.0)$

$0(0.0)$

1 (1.0)

$0(0.0)$

$0(0.0)$

0.190

0.190

0.071

0.507

0.425

1.00

1.00

1.00

IVa

$$
\text { IVb }
$$

Readmission, N (\%)

Refractory Postop

Pain, N(\%)

7 (7.0)

2 (2.0)

0.360

0.096

Table 4: Predictors of Postoperative Complications

\begin{tabular}{lcc}
\hline & Adjusted OR (95\% Cl) & $\boldsymbol{p}$ \\
\hline Charlson Comorbidity Score & $1.47(1.19-1.85)$ & $<\mathbf{0 . 0 0 1}$ \\
Severity & $1.01(0.38-2.55)$ & 0.998 \\
ACS Model & $0.78(0.28-2.16)$ & 0.619 \\
Time to OR (hours) & $1.00(0.99-1.01)$ & 0.723 \\
Operative Approach & $13.09(2.12-121.66)$ & $\mathbf{0 . 0 1 0}$ \\
Operative Duration (min.) & $1.00(0.99-1.01)$ & 0.717
\end{tabular}

\section{Results}

- Baseline population characteristics similar between ACS and non-ACS models

- TTOR and LOS improved with ACS model on univariate analysis but LOS not affected by ACS on multivariate analysis

- No difference in operation length or conversion rates

- No difference in perioperative complication rates

\section{Concusion}

ACS service is effective in decreasing TTOR but has not been shown to decrease LOS. There may be a slight trend favouring ACS in terms of perioperative complications and readmission rates, but no strong evidence to support this currently in Hamilton 